\title{
Synapse Number and Synaptic Efficacy Are Regulated by Presynaptic cAMP and Protein Kinase A
}

\author{
David W. Munno, David J. Prince, and Naweed I. Syed \\ Respiratory and Neuroscience Research Groups, Faculty of Medicine, University of Calgary, Calgary, Alberta, T2N 4N1 Canada
}

\begin{abstract}
The mechanisms by which neurons regulate the number and strength of synapses during development and synaptic plasticity have not yet been defined fully. This lack of fundamental knowledge in the fields of neurodevelopment and synaptic plasticity can be attributed, in part, to compensatory mechanisms by which neurons accommodate for the loss of function in their synaptic partners. This is generally achieved either by scaling up neuronal transmitter release capabilities or by enhancing the postsynaptic responsiveness. Here, we demonstrate that regulation of synaptic strength and number between identified Lymnaea neurons visceral dorsal 4 (VD4, the presynaptic cell) and left pedal dorsal 1 (LPeD1, the postsynaptic cell) requires presynaptic activation of a cAMP-PKA-dependent signal. Experimental activation of the cAMP-PKA pathway resulted in reduced synaptic efficacy, whereas inhibition of the cAMP-PKA cascade permitted hyperinnervation and an overall enhancement of synaptic strength. Because synaptic transmission between VD4 and LPeD1 does not require a cAMP-PKA pathway, our data show that these messengers may play a novel role in regulating the synaptic efficacy during early synaptogenesis and plasticity.
\end{abstract}

Key words: synapse formation; synaptic plasticity; cAMP; PKA; trophic factors; Lymnaea; cell culture

\section{Introduction}

On contact, developing presynaptic and postsynaptic neurons direct their respective synaptic machinery, including neurotransmitter receptors, synaptic vesicles, and synaptic proteins, to the site of newly forming synapses (Ziv and Garner, 2001; Ahmari and Smith, 2002; Garner et al., 2002). Although the processes underlying the differentiation of presynaptic and postsynaptic specializations during synapse formation have been studied extensively at the neuromuscular junction (Sanes and Lichtman, 1999) and in the CNS (Kim and Huganir, 1999; Ziv and Garner, 2001; Ahmari and Smith., 2002; Garner et al., 2002), the mechanisms by which both synapse numbers (i.e., the number of sites that support transmission) and synaptic efficacy (i.e., amplitude of synaptic potentials) are regulated during synaptogenesis and synaptic plasticity remain largely unknown. A fundamental question is: What signals instruct any given presynaptic neuron that its synaptic efficacy has reached full strength with a particular synaptic target during both synapse formation and synaptic plasticity?

One hypothesis is that synaptic efficacy may be regulated globally by enhancing the function of all synapses made by a presynaptic neuron (Turrigiano et al., 1998; Leslie et al., 2001). A second possibility is that synaptic efficacy may be modulated locally at specific synaptic contact sites (Martin et al., 1997a; Casadio et al., 1999). Regulation of synaptic efficacy in either case could be achieved by modulating presynaptic or postsynaptic intracellular signaling pathways (Martin et al., 1997a; Davis et al., 1998), pre-

\footnotetext{
Received Jan. 6, 2003; revised Feb. 19, 2003; accepted Feb. 26, 2003.

This work was supported by the Canadian Institutes of Health Research. N.I.S. is an Alberta Heritage Foundation for Medical Research (AHFMR) Senior Scholar; D.W.M. was supported by a studentship award from AHFMR. Excellent technical support by Wali Zaidi is also acknowledged.

Correspondence should be addressed to Dr. Naweed I. Syed, Department of Cell Biology and Anatomy, Faculty of Medicine, University of Calgary, Calgary, Alberta, Canada T2N 4N1. E-mail address: nisyed@ucalgary.ca. Copyright $\odot 2003$ Society for Neuroscience $\quad$ 0270-6474/03/234146-10\$15.00/0
}

synaptic vesicle release (Davis and Goodman, 1998a), or postsynaptic receptor trafficking or function (Davis and Goodman, 1998a; Colledge et al., 2000; Davis and Bezprozvanny, 2001; Malinow and Malenka, 2002). Because access to all potential synaptic sites at the level of individual presynaptic and postsynaptic neurons in any given preparation is difficult to achieve unequivocally, the precise mechanisms that regulate synaptic efficacy remain unknown.

To define the precise nature of cell-to-cell signaling that regulates synaptic efficacy between central neurons, we took a novel approach in which a single, identified presynaptic neuron was isolated from the Lymnaea CNS and challenged with two identical, supernumerary postsynaptic targets. This model enabled us to study changes in the amplitude of synaptic potentials between individual presynaptic and postsynaptic partners as a physiological index, which represents alterations in synapse efficacy. Specifically, when a single presynaptic neuron, called visceral dorsal 4 (VD4), was paired in a soma-soma configuration with its synaptic partner left pedal dorsal one (LPeD1), of which there is only one cell per animal, excitatory synapses formed in all instances. However, when challenged with two LPeD1 targets, only one postsynaptic cell was innervated by VD4 12-18 hr after cell pairing. When examined $4 \mathrm{hr}$ after pairing, we found that both postsynaptic targets were innervated; however, the total synaptic efficacy was now shared between the two targets. We demonstrate further that extrinsic trophic factors present in the brain conditioned medium (CM) regulate the number of synaptic outputs with postsynaptic targets. This regulation of synaptic efficacy required presynaptic activation of a cAMP-PKA-dependent signal. Experimental activation of the CAMP-PKA pathway resulted in reduced synaptic efficacy, whereas inhibition of the cAMP-PKA cascade permitted hyperinnervation and an overall enhancement of synaptic strength. We propose that a presynaptic, cAMP-PKAdependent signal plays a novel role in regulating synaptic "gain" 
by exerting precise control over synaptic efficacy and allows the innervation of supernumerary targets.

\section{Materials and Methods}

Animals. Laboratory-raised stocks of Lymnaea stagnalis were maintained at room temperature in well aerated pond water and fed lettuce and fish food. Animals used for cell isolation had shell lengths of $18-20 \mathrm{~mm}$ ( $\sim 2-3$ months old), whereas CM was produced from snails that were $\sim 25-30 \mathrm{~mm}$ in length (3-4 months old).

Cell culture. Cell isolation and culture procedures were adapted from those described previously (Syed et al., 1999). Briefly, the central ring ganglia were isolated and washed with antibiotic saline (in mM: 51.3 $\mathrm{NaCl}, 1.7 \mathrm{KCl}, 4.0 \mathrm{CaCl}_{2}$, and $1.5 \mathrm{MgCl}_{2}$ buffered with HEPES to a $\mathrm{pH}$ of 7.9 and added gentamicin, $50 \mu \mathrm{g} / \mathrm{ml}$ ) three times for $15 \mathrm{~min}$. Ganglia were subsequently treated with trypsin $(2 \mathrm{mg} / \mathrm{ml}$, Sigma, St. Louis, MO) and then trypsin inhibitor $(2 \mathrm{mg} / \mathrm{ml}$, Sigma), each dissolved in $50 \%$ Liebowitz L-15 (Invitrogen, San Diego, CA, special order) defined medium (DM). DM was prepared with added inorganic salts (same concentration as saline) and $20 \mu \mathrm{g} / \mathrm{ml}$ gentamicin (Syed et al., 1999).

The central ring ganglia and buccal ganglia were pinned down on a dissection dish containing high-osmolarity DM (DM with added $20 \mathrm{~mm}$ D-glucose). Neurons were isolated and extracted using a Gilmont syringe and fire-polished glass pipettes (50-90 $\mu \mathrm{m}$ tip diameter). Isolated cells were plated in CM onto glass coverslips pretreated with poly-L-lysine (MW 95,000) that were attached to plastic dishes (Falcon 3001) or on coverslips that were pretreated with Lymnaea hemolymph (pretreatment of glass coverslips with Lymnaea hemolymph prevents cells from adhering to the dishes).

To prepare CM, gentamycin-treated ganglia were incubated in DM contained in Sigma-Cote (Sigma)-treated glass Petri dishes for $72 \mathrm{hr}$ (in a humidified chamber). The isolated ganglia were removed, and the $\mathrm{CM}$ was frozen at $-20^{\circ} \mathrm{C}$ until use (up to 6 months). Although the specific composition of various neurotrophic proteins present in the CM is unknown, previous studies have identified at least two Lymnaea trophic molecules that partially mimic the effects of CM on neurite outgrowth [cystine-rich neurotrophic factor (Fainzilber et al., 1996); Lymnaea epidermal growth factor (Hermann et al., 2000)] and synapse formation [Lymnaea epidermal growth factor (Hamakawa et al., 1999)].

Lymnaea hemolymph was drawn from the foot by use of sterile hypodermic needles and syringes and used to coat dishes immediately. The filtered ( $0.2 \mu \mathrm{m}$, Millipore) hemolymph was added to the glass coverslips $(\sim 1 \mathrm{ml} /$ dish $)$ and left for $30 \mathrm{~min}$. The hemolymph was then removed, the dishes were allowed to dry for $30 \mathrm{~min}$, and the coverslips were subsequently washed with sterile water. Before neuronal plating, $\mathrm{CM}$ was added to hemolymph-pretreated dishes. Neurons plated on hemolymph-pretreated dishes were left for $12-18 \mathrm{hr}$ to prevent cell adhesion and then transferred to poly-L-lysine coated dishes.

To inhibit PKA activity, presynaptic neurons were incubated selectively in H-89 (Calbiochem, La Jolla, CA) (Zhang et al., 2002) or KT5720 (Calbiochem) (Diefenbach et al., 2000), which were dissolved in DMSO (final [DMSO] $<0.01 \%$ ). 8Br-cAMP (Research Biochemicals, Natick, $\mathrm{MA}$ ) and acetylcholine were dissolved in water. All chemicals were obtained from Sigma unless stated otherwise.

Electrophysiology. Conventional electrophysiological techniques were used to record simultaneous intracellular activity from pairs of neurons as described previously (Feng et al., 1997; Woodin et al., 2002). Specifically, neurons were viewed under a Zeiss Axiovert 135 (Zeiss, Oberkochen, Germany) inverted microscope and impaled using Narishige (Tokyo, Japan) micromanipulators (MM 202 and MM 204). Glass microelectrodes were filled with a saturated solution of potassium sulfate $\left(\mathrm{K}_{2} \mathrm{SO}_{4}\right.$; electrode resistance, $20-40 \mathrm{M} \Omega$ ). Intracellular signals were amplified (IR-283; Neurodata Instruments, New York, NY), displayed on a storage oscilloscope (Fluka 2000, Buchs, Switzerland), and recorded digitally using a digitizer (Digidata 1200; Axon Instruments, Union City, CA) and acquisition software (Clampex version 7.0; Axon Instruments). Data analyses were performed using Clampfit version 8.0 (Axon Instruments) and SigmaPlot 4.0 (Jandel Scientific, SPSS Science, Chicago, IL). Results are presented as mean \pm SEM.

To test synaptic ACh sensitivity, a modified patch pipette (5-8 M $\Omega$ ) was manipulated in close proximity to the postsynaptic neuron at the site of contact between the paired cells. ACh was pressure-applied (1-2 psi) using $1 \mathrm{sec}$ pulses. Using this paradigm, we have shown previously that ACh application at the contact site (synaptic) induces significantly larger postsynaptic responses compared with noncontact (extrasynaptic) sites (Meems et al., 2003).

FM1-43 loading and imaging. FM1-43 loading and imaging procedures were adapted from those described previously (Murthy et al., 2001). FM1-43 was added to the bath at a final concentration of $2.5 \mu \mathrm{M}$. After a 10 min incubation with the dye, the presynaptic cell (VD4) was stimulated (80-120 action potentials) by injecting depolarizing current via sharp intracellular electrodes. After $5 \mathrm{~min}$ had been allowed for the uptake of FM1-43, the preparation was washed thoroughly with cold $\left(4^{\circ} \mathrm{C}\right)$ normal saline for $10 \mathrm{~min}$ before replacing the culture media with normal saline at room temperature. Fluorescent images were acquired using a Zeiss Axiovert 200M inverted microscope equipped with $20 \times[0.50 \mathrm{nu}-$ merical aperture (NA)] and $40 \times(0.75 \mathrm{NA})$ objectives (Plan Neofluar). Excitation light was from a $100 \mathrm{~W}$ mercury lamp, excitation filters (480/40 or 535/50 nm), dichroic mirror (505 or $565 \mathrm{~nm}$ ), and emission filters $(535 / 50$ or $610 / 75 \mathrm{~nm})$. Images were captured with a Photometrics (Tucson, AZ) SenSys 1400 camera (100-300 msec exposure) connected to a computer running Axiovision 3.0 for Windows (Carl Zeiss).

Experimental paradigm. Each Lymnaea central ring ganglion (CRG) contains one VD4 and LPeD1. In vivo and in vitro, VD4 forms a one-way excitatory synapse with LPeD1 (Hamakawa et al., 1999; Syed et al., 1999; Woodin et al., 1999). The identified presynaptic neuron VD4 was paired with its postsynaptic partner LPeD1. When paired in CM, VD4 established its excitatory synapse with LPeD1 in all instances. When VD4 was paired simultaneously with two LPeD1 cells isolated from two different animals, the cell that received excitatory innervation from VD4 (the appropriate, in vivo synapse), as determined electrophysiologically, was designated LPeDla, whereas the uninnervated LPeD1 was called LPeD1b. In other experiments, the first LPeD1 that was paired with VD4 was designated LPeD1a, and the second LPeD1 that was paired with VD4 was labeled LPeD1b.

Statistical analyses. Data are presented as mean \pm SEM. Statistical analysis was performed using Sigmastat (Jandel Scientific). Differences between mean values from each experimental group were tested by use of a Student's $t$ test for two groups and one-way ANOVA for more than two groups and were considered significant if $p<0.05$.

\section{Results}

We have demonstrated previously that Lymnaea presynaptic neurons recapitulate their specific synapses with appropriate target cells both in cell culture (Syed et al., 1990; Feng et al., 1997; Hamakawa et al., 1999; Woodin et al., 1999, 2002) and after single-cell transplantation (Syed et al., 1992). The efficacy of synaptic transmission remains consistent in vitro between both soma-soma and neurite-neurite paired cells (Syed et al., 1990; Feng et al., 1997) and in vivo after single-cell transplantation into the intact ganglia (Syed et al., 1992). To gain direct access to both presynaptic and postsynaptic somata and their synaptic sites, we took advantage of the soma-soma preparation in which specific synapses were reconstructed between the cell bodies of synaptic partners (Feng et al., 1997). The soma-soma synapses have been shown previously to be target-cell-specific, require gene transcription and de novo protein synthesis, and are both ultrastructurally and electrophysiologically similar to those observed either in vivo or in neurite-neurite configuration (Syed et al., 1990, 1992; Feng et al., 1997; Hamakawa et al., 1999; Woodin et al., 1999, 2002; Smit et al., 2001). Because synapses between somasoma pairs develop within hours of pairing, this preparation also enabled us to regulate the precise timing at which the cell-cell interactions can be initiated. 
VD4 does not innervate supernumerary targets

To test the ability of a single presynaptic neuron to innervate supernumerary targets, VD4 was isolated and paired simultaneously with two LPeD1 cells, each from a different CRG (VD4 was isolated from a different CRG than either postsynaptic target). The cell bodies were juxtaposed in a triplet configuration (Fig. 1 $A$ ) in the presence of CM. Approximately 12-18 hr after the neurons had been juxtaposed, pairwise intracellular recordings revealed that VD4 had reestablished a synapse with only one of the postsynaptic targets (which were called LPeD1a). Intracellular current injection into VD4 induced 1:1 EPSPs (average EPSP amplitude, $6.12 \pm$ $0.33 \mathrm{mV}$ ) in LPeDla in all experiments $(100 \% ; n=19)$ (Fig. $1 B, C)$. In contrast, 95\% of the supernumerary LPeD1b cells did not receive innervation from VD4 (Fig. 1B,C), although VD4 has been shown previously to simultaneously innervate distinct multiple targets both in vivo (Syed et al., 1992) and in vitro (Syed et al., 1990).

To determine whether presynaptic machinery was localized only to the innervated postsynaptic target, the triplet was incubated with the styryl dye FM1-43 (2.5 $\mu \mathrm{M})$, which is a marker of presynaptic active zones (Murthy et al., 2001; Ahmari and Smith, 2002). FM1-43 was bathapplied, and VD4 cells were loaded with the dye by evoking trains of action potentials via an intracellular electrode. Corresponding to the intracellular recordings, we found that FM1-43-labeled synaptic terminals were localized exclusively at one postsynaptic target, whereas no detectable presynaptic vesicle recycling was observed at the contact site with the other postsynaptic neuron $(n=10)$ (Fig. $1 D)$. These data are consistent with electrophysiological observations, and together, they suggest that VD4 innervates only one of the two simultaneously paired LPeD1 cells.

To rule out the possibility that the excluded postsynaptic cells were somehow incapable of synaptogenesis, an additional VD4 (VD4b) was juxtaposed against a previously uninnervated LPeD1b. Simultaneous intracellular recordings made both 4 and 12-18 hr after pairing revealed that a train of action potentials in VD4b induced a 1:1 excitatory postsynaptic response in LPeD1b $(n=5)$ (Fig. $1 E)$. These data demonstrate that LPeD1b was indeed healthy and synaptically viable.

\section{A critical period for establishing synapses with multiple targets}

The above data do not rule out the possibility that VD4 may have innervated both postsynaptic targets simultaneously at some
B
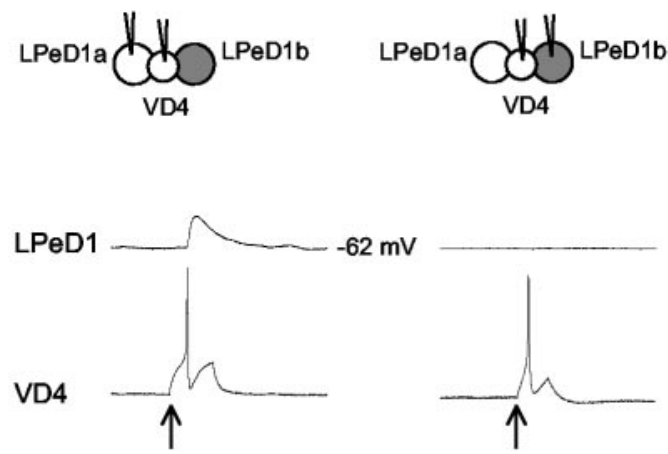

C (19)
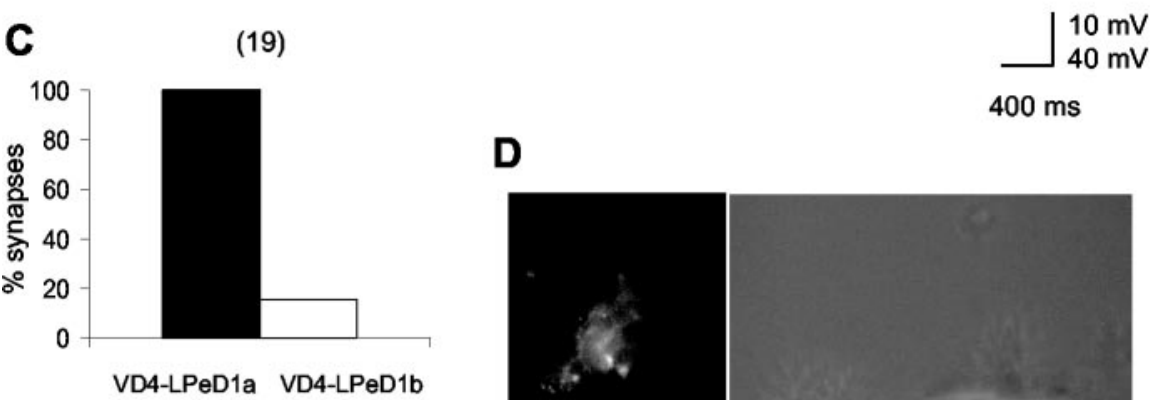

E

\section{D} $400 \mathrm{~ms}$

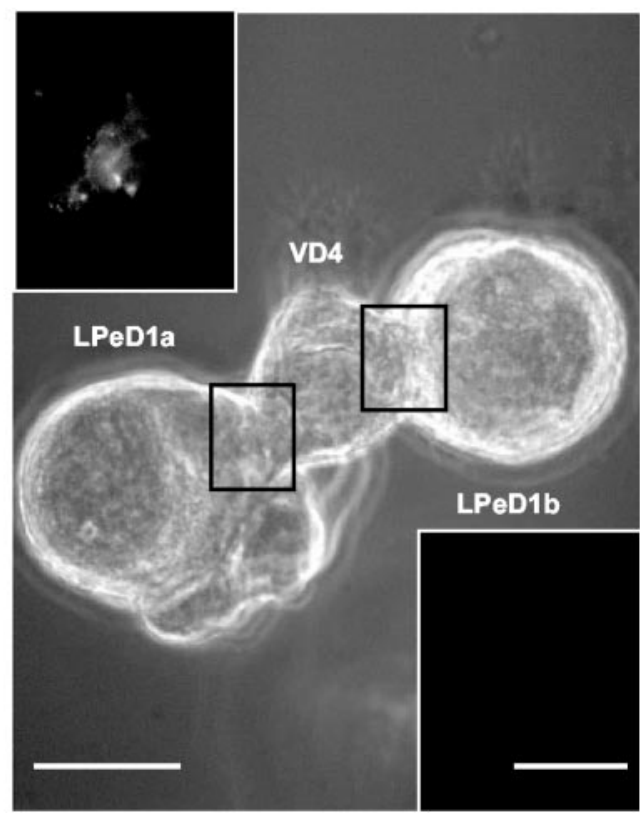

Figure 1. VD4 does not innervate supernumerary targets. Identified Lymnaea neurons were isolated in cell culture and paired overnight as soma-soma triplets. Intracellular recordings were made to demonstrate chemical synapses between the paired cells. A, Photomicrograph of a VD4 that was soma-soma paired with two LPeD1 cells. B, Representative traces from pair-wise intracellular recordings of soma-soma triplets. Induced action potentials in VD4 generated 1:1 EPSPs in LPeD1a but not in LPeD1b. $C$, Summary data showing the percentage of LPeD1a and LPeD1b that formed excitatory synapses with VD4 in CM. D, Phase-contrast picture of soma-soma triplet that was incubated in FM1-43 dye. VD4 was stimulated intracellularly for 2-3 min by injecting pulses of depolarizing current. After dye washout, fluorescent images of FM1-43 loaded cells were acquired. Insets show pseudo-color images of FM1-43, which was localized exclusively in VD4 at its contact site with only one (LPeD1a, top inset) but not the other LPeD1 cell (LPeD1b, bottom inset).E, A photomicrograph depicting cells paired in quadruplet configuration. Specifically, a second VD4 (VD4b) was juxtaposed against a previously uninnervated LPeD1b from a soma-soma triplet. Representative intracellular recordings made $4 \mathrm{hr}$ after juxtaposing the VD4b demonstrate that action potentials in the VD4b cell generate 1:1 EPSPs in LPeD1b. Scale bars: phase-contrast images, $50 \mu \mathrm{m}$; fluorescent images, $25 \mu \mathrm{m}$.

time point during early synapse formation, one of which was subsequently retracted when tested $12-18 \mathrm{hr}$ after pairing. Therefore, to determine the precise temporal pattern of innervation, VD4 and two LPeD1 neurons were isolated separately and paired systematically, and synapses were tested shortly after pairing. Previous studies have shown that the development of synapses between VD4 and its postsynaptic targets occurs within $4 \mathrm{hr}$ after pairing in a soma-soma configuration (Woodin et al., 2002). In the first set of experiments, VD4 was paired simultaneously with both LPeD1 neurons. Pairwise intracellular recordings made $4 \mathrm{hr}$ 
A
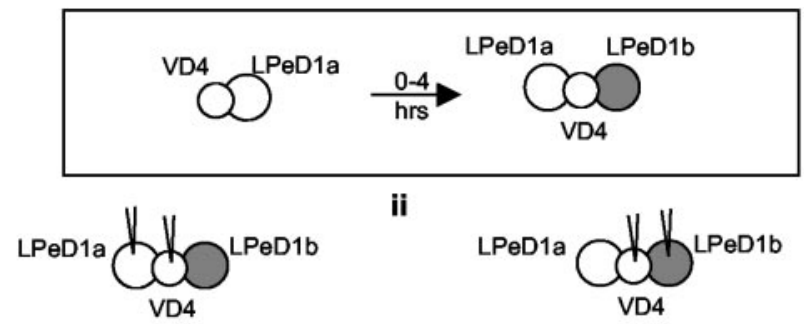

ii
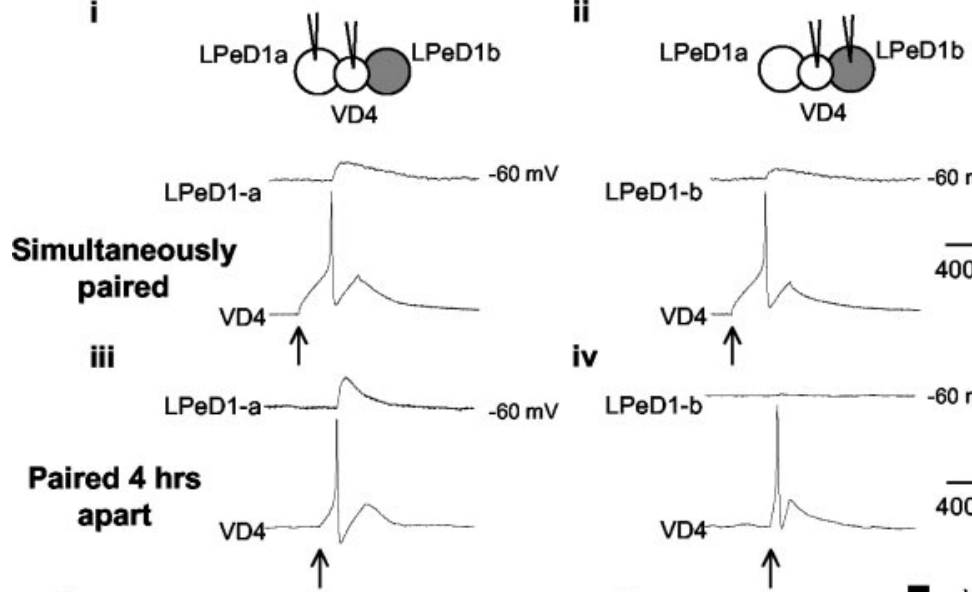

B
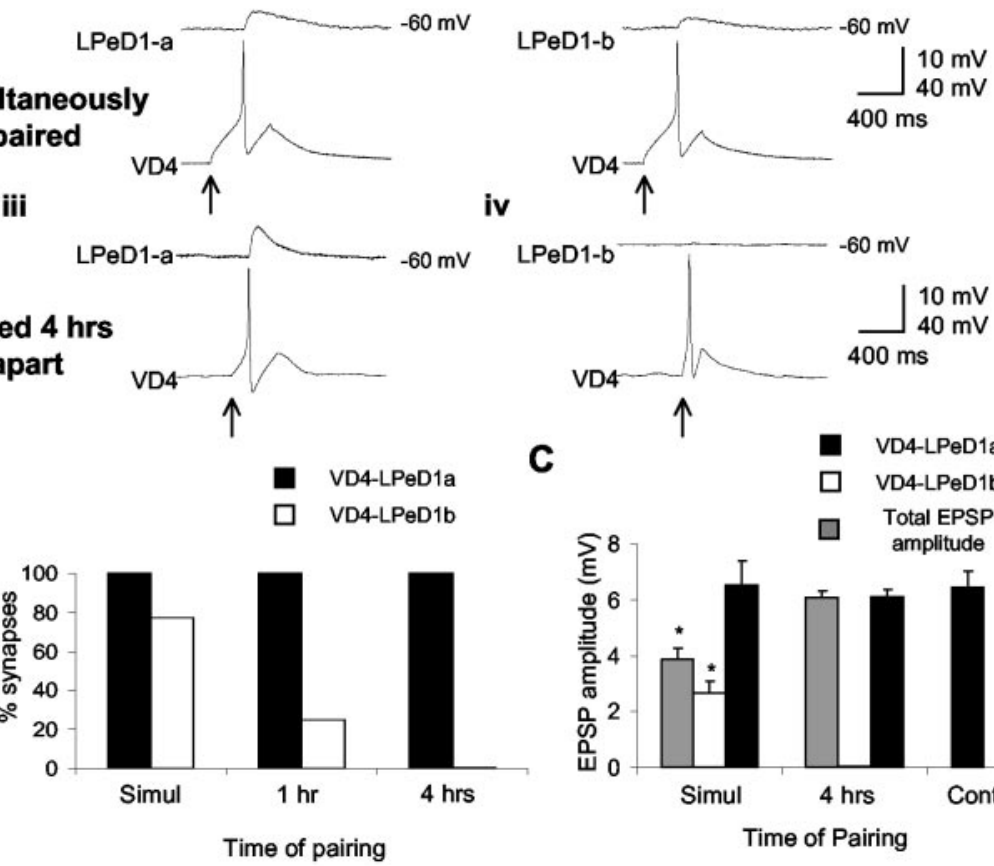

(1)

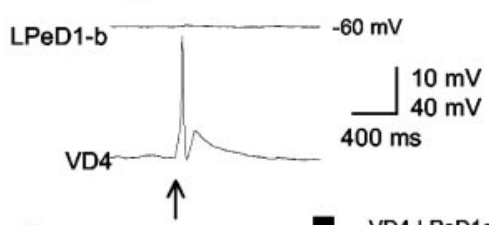

C

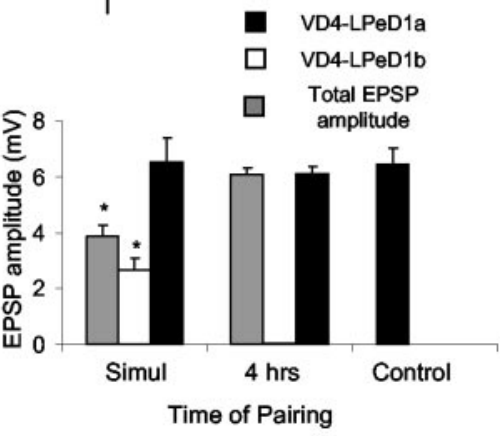

D

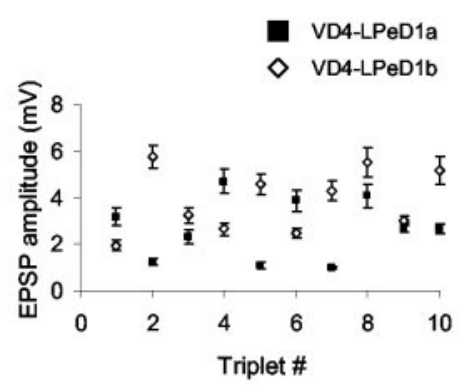

Simultaneously paired
E

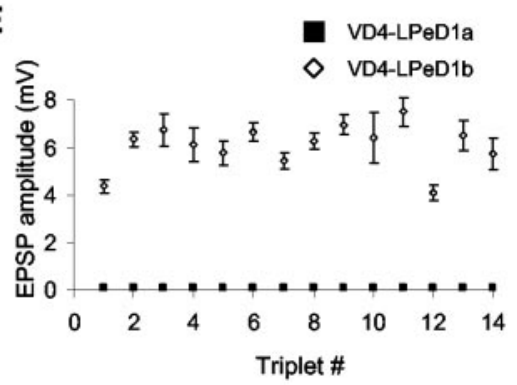

Paired 4 hrs apart

Figure 2. Innervation patterns and synaptic efficacy between VD4 and LPED1 cells is temporally regulated. To define the precise pattern of synaptic innervation between VD4 and multiple LPeD1 cells, these neurons were juxtaposed against VD4 at different time points. Top (box), experimental paradigm. VD4 was paired with LPeD1a, and the second LPeD1 (LPeD1b) was brought into contact either simultaneously or 1 or $4 \mathrm{hr}$ after contact with the first cell. Pairwise intracellular recordings were made $4 \mathrm{hr}$ after LPeD1b was juxtaposed. When juxtaposed simultaneously, both LPeD1 cells ( $a$ and $b$ ) were innervated by VD4. Induced action potentials in VD4 generated 1:1 EPSPs in both cells (Ai, Aii). However, when LPeD1b was juxtaposed with VD4 4 hr after LPeD1a, a synapse was detected only in LPeD1a (Aiii, Aiv). B, C, Summarized data of the percentage of synapses formed ( $B$ ) and absolute EPSP amplitude with each target ( $C$. D, E, Average EPSP amplitude recorded in each postsynaptic target in triplet configuration paired simultaneously $(D)$ or after a $4 \mathrm{hr}$ delay $(E) . D$, EPSP data are shown only for those triplets in which a synapse was formed with both targets.

after the cells were paired revealed that in $77 \%$ of the cases $(n=$ 13), VD4 had formed excitatory synapses with both LPeD1 targets (Fig. $2 A, B$ ). Because synapses were formed with both targets, the LPeD1 that was tested first was designated LPeDla, whereas the second cell was called LPeD1b. To test whether the synaptic efficacy between VD4 and both LPeD1 cells was similar to that of a control pair (VD4 and a single LPeD1 recorded $4 \mathrm{hr}$ after pair- ing), the EPSP amplitude was recorded from both LPeD1 cells and measured during a series of 10 action potentials. We found that the efficacy of synaptic transmission between VD4 and the two LPeD1 cells, paired in a triplet configuration, was significantly weaker with each postsynaptic target (LPeDla average EPSP amplitude, $3.85 \pm 0.434 \mathrm{mV}, p<0.0001$, oneway ANOVA; LPeD1b average EPSP amplitude, $2.67 \pm 0.411 \mathrm{mV}, p<0.0001$, one-way ANOVA) compared with control pairs (VD4 with only one $\mathrm{LPeD} 1 ; n=$ 11; $6.43 \pm 0.575 \mathrm{mV}$ ) (Fig. $2 C-E$ ). The EPSP amplitude recorded from single VD4-LPeD1 pairs after $4 \mathrm{hr}$ was not significantly different from that after 12-18 hr $(n=19 ; 6.12 \pm 0.33 \mathrm{mV})$. Interestingly, although the synaptic strength of each target under the above experimental conditions was weaker than normal, the sum of combined synaptic strength between both targets $(6.52 \pm 0.845 \mathrm{mV})$ was not significantly different from the control pairs $(p=0.35 ; t$ test) (Fig. $2 C$ ). These data demonstrate that (1) during early stages of synapse formation, VD4 is capable of innervating supernumerary targets and (2) the sum of total synaptic strength is tightly regulated by VD4. Therefore, supernumerary innervation is achieved at the expense of total synaptic strength.

We next sought to determine whether one LPeD1, given a head start, would "capture" innervation from VD4 with full synaptic strength. VD4 was thus paired immediately with one LPeD1, whereas the second LPeD1 was brought in contact either $1 \mathrm{hr}(n=8)$ or $4 \mathrm{hr}(n=14)$ after pairing with the first LPeD1. When the second LPeD1 was brought into contact with VD4 after a 1-hr delay, 25\% of these cells established excitatory synapses with VD4 (Fig. 2B). For the two pairs in which both postsynaptic targets formed synapses with VD4, the sum of the combined synaptic strength between the two targets (LPeDla average EPSP amplitude, $4.1 \pm$ $0.4 \mathrm{mV}$; LPeD1b average EPSP amplitude, $2.4 \pm 0.3 \mathrm{mV}$; combined EPSP amplitude, $6.50 \pm 0.7 \mathrm{mV}$ ) was not significantly different from that of control pairs. In contrast, the second LPeD1 was always excluded from the synaptogenic program when juxtaposed $4 \mathrm{hr}$ after the first target was paired with VD4 (Fig. $2 A i v, B$ ). In this triplet configuration, the EPSP amplitude of the innervated LPeD1 $(6.06 \pm 0.246 \mathrm{mV})$ was not significantly different from that of control pairs (Fig. $2 C, E)$. These data suggest that a critical period exists during which multiple, identical targets may be innervated. However, when synapses with one postsynaptic target reach full strength, the synaptogenic program stops and the supernumerary targets 
are systematically excluded. Furthermore, these data suggest that the synaptic strength may be the determining factor that mediates the exclusion signal.

\section{Trophic factors modulate the number} of synaptic connections

VD4 is known to form both excitatory and inhibitory synapses with defined postsynaptic targets in vivo (Syed et al., 1990; Syed and Winlow, 1991). Moreover, both excitatory and inhibitory synapses between VD4 and its postsynaptic targets in cell culture have been shown to be cholinergic (Woodin et al., 2002). Although trophic factors are required for excitatory (Hamakawa et al., 1999; Woodin et al., 1999, 2002) but not inhibitory (Feng et al., 1997) synapse formation between cultured Lymnaea neurons, the trophic factor-induced excitatory synaptogenesis does not involve a switch (from cholinergic to other transmitters) in the presynaptic phenotype (Woodin et al., 2002). In this study, we next asked whether extrinsic trophic factors regulate innervation of supernumerary targets or synaptic efficacy between VD4 and LPeD1. VD4 was isolated and juxtaposed with two LPeD1 targets in DM, which lacks trophic factors. Approximately $12-18 \mathrm{hr}$ after pairing, the neurons were impaled with sharp electrodes, and synapses were tested electrophysiologically. Interestingly, VD4 established novel, inappropriate inhibitory synapses (synapse in vivo is excitatory) with both LPeD1 cells (94\%; $n=17$ ) (Fig. $3 A-C)$. Evoked action potentials in VD4 induced 1:1 (Fig. $4 A, B$ ) or compound IPSPs in both postsynaptic LPeD1 neurons. For the eight triplets in which VD4 induced unitary IPSPs in both LPeD1 cells, the amplitude of 1:1 IPSPs for both postsynaptic neurons in triplets (LPeD1a: IPSP amplitude, $-3.55 \pm 0.335 \mathrm{mV}$; LPeD1b: IPSP amplitude, $-3.64 \pm 0.349 \mathrm{mV}$ ) was not significantly different from those in single pairs (LPeD1 pairs: IPSP amplitude, $-4.14 \pm 0.154 \mathrm{mV}$; $n=7$ ) (Fig. 3D). However, in contrast to triplets cultured in CM, the sum of combined synaptic strength between both targets paired in DM in triplet configuration $(-7.19 \pm 0.309 \mathrm{mV})$ was significantly stronger from the control pairs $(p<0.0001 ; t$ test). These data suggest that trophic factors not only are required for excitatory synapse formation but also are needed to implement the exclusion signal. Moreover, these data suggest that the "exclusion" rule does not apply to default inhibitory synapses described previously (Woodin et al., 1999).

\section{cAMP and PKA control presynaptic regulation of synapse number and efficacy}

cAMP and cAMP-dependent protein kinases (PKA) have been implicated in structural and functional changes during synaptic plasticity (Huang et al., 1994; Weisskopf et al., 1994; Salin et al., 1996; Martin et al., 1997b; Mellor et al., 2002). Specifically, in both vertebrates and invertebrates, increases in cAMP levels or an
B

C

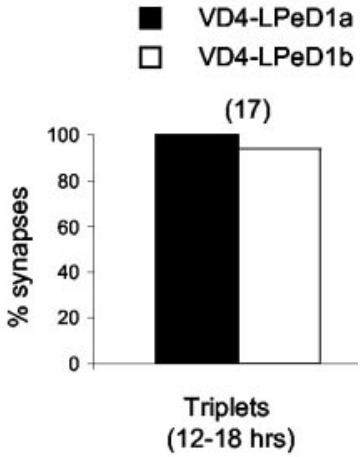

$\mathbf{E}$

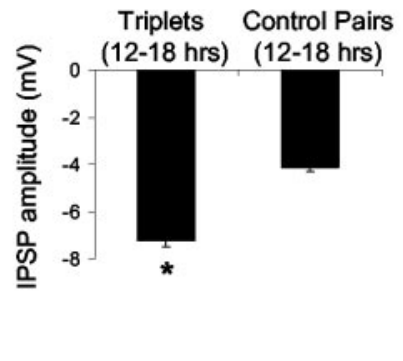

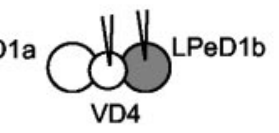

$-62 \mathrm{mV}$

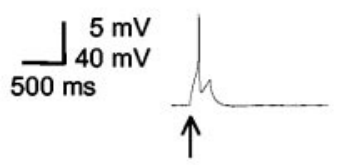

(12-18 hrs)

Figure 3. The exclusion of supernumerary target innervation by VD4 is contingent on CM-derived trophic factors. To test whether trophic factors are required for the mediation of exclusion signal, cells were paired in DM. Simultaneous intracellular (

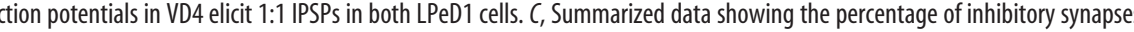

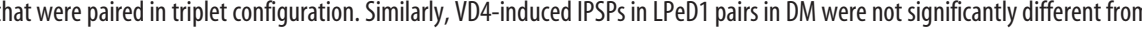
PPS observed in either LPeD1 cell in triplets. E, Summary data for the combined IPSP amplitude of both postsynaptic targets in DM-treated triplets compared with the IPSP amplitude of control pairs. All IPSPs were measured at a postsynaptic membrane potential of $-60 \mathrm{mV}$. Arrows indicate the injection of depolarizing current.

enhancement of PKA activity lead to new synaptic growth and a long-term enhancement of synaptic strength (Martin and Kandel, 1996; Martin et al., 1997). To test for the involvement of cAMP and PKA in regulating the synaptic strength during synapse formation, we took advantage of soma-soma paired neurons. cAMP levels and PKA activity were manipulated pharmacologically in either the presynaptic or postsynaptic cells before soma-soma pairing. The effects of these pharmacological manipulations on synaptic efficacy were tested electrophysiologically. Specifically, we incubated the presynaptic VD4 neurons for 12-18 hr in CM containing 8Br-cAMP (cAMP analog/PKA agonist). VD4 neurons were subsequently washed with DM and paired with a single LPeD1 in the presence of normal CM. Selective enhancement of cAMP-PKA activity in the presynaptic neuron decreased synaptic efficacy between VD4 and LPeD1 pairs in a dose-dependent manner (Fig. 4). EPSP amplitude was reduced to approximately half of control values in the presence of $100 \mu \mathrm{M}$ $8 \mathrm{Br}$-cAMP. This result is in contrast to the previously ascribed role of cAMP in the enhancement of synaptic growth and strength (Martin and Kandel, 1996; Martin et al., 1997).

Although the efficacy of synaptic transmission decreased after selective pretreatment of the presynaptic neuron with PKA ago- 
A

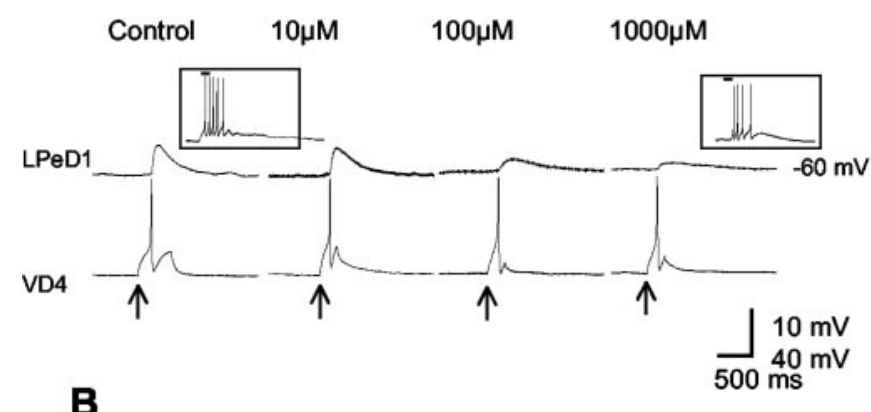

B

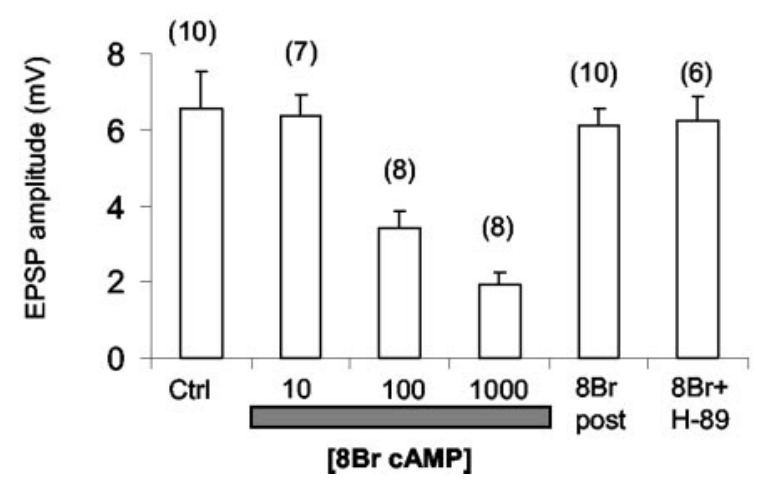

Figure 4. Presynaptic activation of cAMP pathway decreases synaptic efficacy at VD4 and LPeD1 synapses. To test whether CAMP-dependent pathway determines the efficacy of synaptic strength, VD4 was paired with LPeD1 in either absence or presence of 8Br-cAMP. Before intracellular recordings, the drug was washed with normal DM, and the efficacy of synaptic strength was tested. Compared with their control counterparts, increasing concentrations of 8Br-CAMP $(10-1000 \mu \mathrm{M})$ reduced synaptic efficacy between VD4 and LPeD1. A, Representative traces of intracellular recordings from VD4 and LPeD1. In normal CM, VD4 induced 1:1 EPSPs in LPeD1. However, when cells were maintained overnight in $\mathrm{CM}$ containing $8 \mathrm{Br}$-CAMP and subsequently recorded under normal DM conditions, the amplitude of VD4-induced EPSPs decreased in a dose-dependent manner with increasing concentrations of the cAMP analog. To rule out the possibility that postsynaptic responsiveness to ACh (VD4 transmitter) did not change after chronic treatment with $8 \mathrm{Br}$-CAMP, synaptic ACh sensitivity in $\mathrm{LPeD1}$ was tested by exogenously applying ACh to LPeD1 specifically at the contact site with VD4. The response to exogenous ACh was similar in both control and $1000 \mu \mathrm{M}$ 8Br-cAMP (insets). B, Summary data showing the average EPSP amplitude for all experiments in each condition. Selective treatment of the postsynaptic cell with 8Br-cAMP did not affect EPSP amplitude. Moreover, the reduction of synaptic efficacy in the presence of CAMP analogs was blocked when the presynaptic neurons were coincubated with $8 \mathrm{Br}-\mathrm{CAMP}$ and $\mathrm{H}-89$.

nists, the postsynaptic responsiveness to exogenously applied acetylcholine (ACh; $1 \mu \mathrm{M}$ ), which is the neurotransmitter used at the VD4-LPeD1 synapse, did not change. Specifically, using a modified patch pipette, ACh was applied exogenously (1 sec pulses) at the site of contact between the paired cells (Meems et al., 2003). Exogenously applied ACh induced comparable depolarizing responses that led to action potentials in LPeD1 neurons from pairs in all treatment conditions (Fig. $4 A$, insets; shown for control and $1000 \mu \mathrm{M}$ cAMP). Similarly, selective treatment of the postsynaptic cell with $8 \mathrm{Br}$-cAMP $(1000 \mu \mathrm{M})$ and subsequent pairing with VD4 did not alter synaptic efficacy between the paired cells ( $n=10$; average EPSP amplitude, $6.10 \pm 0.42 \mathrm{mV})$. However, the 8Br-cAMP-induced reduction in synaptic efficacy was blocked when the presynaptic neurons were coincubated with 8Br-cAMP and the selective PKA antagonist $\mathrm{H}-89$ (200 nM) $(n=6$; average EPSP amplitude, $6.21 \pm 0.67 \mathrm{mV})$ (Fig. $4 B$ ). These data suggest that cAMP and/or PKA activation in the presynaptic neuron may play a novel role during early synaptogen- esis, such that these messengers may regulate the efficacy of synaptic strength between synaptic partners.

PKA antagonists perturb presynaptic regulation of number and efficacy of synapses

To define further the mechanisms that regulate synaptic efficacy, we next attempted to identify the nature of signals that endowed VD4 with the ability to eliminate its extra synapses with supernumerary LPeD1 cells. If cAMP or PKA activation in VD4 reduces the efficacy of synaptic transmission with LPeD1, then presynaptic inhibition of PKA activity should enhance synaptic efficacy. To test this hypothesis directly, VD4 was incubated selectively in CM containing the PKA antagonists H-89 (200 nM) or KT5720 (500 nM) for 12-18 hr. In control pairs, VD4 was paired with a single LPeD1 cell. In the experimental conditions, VD4 was juxtaposed with one LPeD1 (LPeD1a) followed by a second LPeD1 (LPeD1b) $4 \mathrm{hr}$ later, a paradigm in which a synapse is normally formed with only one target (Fig. 2). Pairwise recordings revealed that when PKA activity was blocked selectively in VD4, it successfully innervated both LPeD1 cells (H-89, $n=8$; KT5720, $n=6$ ) (Fig. $5 A, B$ ). Experimental elevation of cAMP using $8 \mathrm{Br}$-cAMP in the presence of $\mathrm{H}-89$, however, did not prevent hyperinnervation of both LPeD1 cells by VD4 $(n=7)$ (Fig. $5 B)$. These data suggest that PKA functions downstream of cAMP and that PKA activity is essential for regulating the number of synapses between the paired cells. To test further whether the regulation of synapse numbers and synaptic efficacy required PKA activity in the presynaptic but not the postsynaptic cell, both LPeD1 neurons were treated selectively with $\mathrm{H}-89$ before pairing and juxtaposed simultaneously with an untreated VD4 neuron. Under these experimental conditions, pairwise recordings revealed that VD4 innervated only one of the postsynaptic neurons in $89 \%$ of the cases $(n=9)$.

To examine further the effect of presynaptic inhibition of PKA activity on synapse number and efficacy, we next measured the efficacy of synaptic strength with both postsynaptic cells in triplets in which the presynaptic neuron was treated with $\mathrm{H}-89$. The efficacy of synaptic strength with both postsynaptic cells (VD4LPeD1a average EPSP amplitude, $6.64 \pm 0.607 \mathrm{mV}$; VD4LPeD1b average EPSP amplitude, $5.97 \pm 0.477 \mathrm{mV}$ ) was not statistically different from that of control $\mathrm{H}$-89-treated single pairs $(6.17 \pm 0.442 \mathrm{mV})$ or untreated control pairs $(6.43 \pm 0.575)$ (Fig. 5C,E). These data suggest that PKA signaling in the presynaptic neuron may participate in the exclusion of a supernumerary target $(\mathrm{LPeD} 1 \mathrm{~b})$.

If PKA is indeed involved in regulating the number and efficacy of synaptic contacts, then disrupting PKA signaling should augment synaptic efficacy between VD4 and LPeD1. Consistent with this hypothesis, the combined synaptic strength in a triplet configuration, in which the presynaptic neuron was pretreated with H-89 was found to be increased to $208 \%$ (12.61 \pm 0.541 $\mathrm{mV}$ ) compared with control-treated triplets (Fig. 5C,D). Together, these data demonstrate that presynaptic cAMP-PKAdependent pathways may regulate synaptic efficacy and the number of synapses during synapse formation.

\section{Presynaptic activation of cAMP rescues inhibitory hyperinnervation in the absence of trophic factors}

Because trophic factors are required for excitatory synapse formation and synaptic efficacy was shown to be regulated by trophic factors and cAMP-PKA-dependent mechanisms, we next asked whether inappropriate inhibitory synapses and synaptic strength observed in DM (Fig. 3) could be rescued by cAMP 
A $\mathrm{i}$

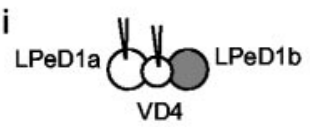

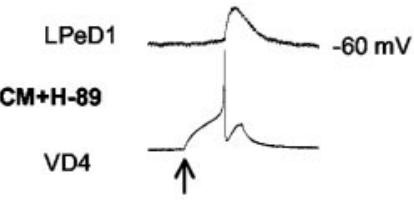

B
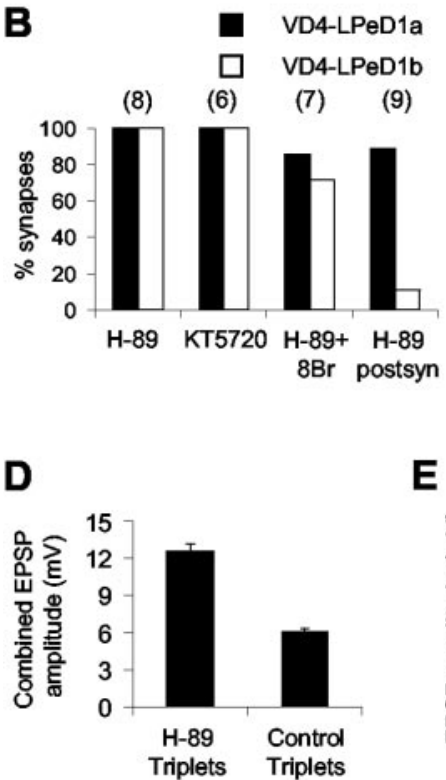

E

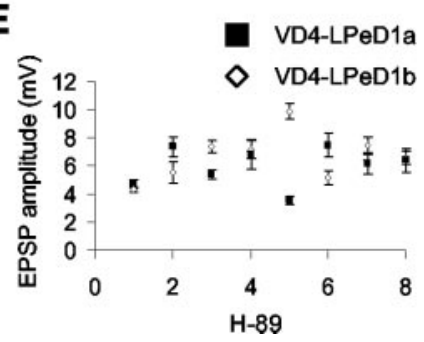

Figure 5. Presynaptic suppression of PKA activity enables hyperinnervation and an enhancement of synaptic efficacy. To test for the involvement of PKA in regulating the synaptic efficacy, cells were paired in the presence of PKA antagonists. Specifically, VD4 was first paired with LPeD1a in the presence of CM + H-89 (PKA antagonist), and, after $4 \mathrm{hr}$, a second LPeD1 was juxtaposed against VD4. After the drug washout, simultaneous intracellular recordings were made from all cells. $A$, Representative traces of pairwise recordings made $4 \mathrm{hr}$ after the second LPeD1 was juxtaposed demonstrate that action potentials in VD4 induced 1:1 EPSPs of equal amplitude in both LPeD1 cells. B, Summary data showing the percentage of LPeD1a and LPeD1b neurons that formed synapses with VD4 when the presynaptic neuron was treated selectively with $\mathrm{H}-89$ or KT5720. Hyperinnervation induced by PKA antagonists was not rescued by the addition of the CAMP analog 8Br-cAMP. In control experiments, VD4 innervated only one postsynaptic target when the postsynaptic neuron was treated selectively with H-89. C, After H-89 pretreatment, the average EPSP amplitude for each LPeD1 neuron, either in a triplet configuration or when paired, is compared with the control cells. No significant difference was observed between VD4-induced EPSPs in both LPeD1 cells that were paired with a single VD4 in the presence of $\mathrm{H}-89$. Similarly, VD4-induced EPSPs in LPeD1 pairs in $\mathrm{H}-89$ was identical to that of control pairs and also to those cultured as triplets in the presence of PKA inhibitor. D, Summary data for the combined EPSP amplitude of both postsynaptic targets in $\mathrm{H}$-89-treated triplets compared with the total EPSP amplitude of control triplets, in which only one postsynaptic target was innervated. E, Scatterplot of the average EPSP amplitude for both postsynaptic targets in $\mathrm{H}$-89-treated triplets.

analogs. Specifically, VD4 was paired simultaneously with two LPeD1 cells in DM in the presence of 8Br-cAMP $(100 \mu \mathrm{M})$. Approximately $12-18 \mathrm{hr}$ after the cells were paired, the drug was washed with normal DM, and intracellular recordings were made. We found that although cAMP analog failed to restore the excitatory phenotype (i.e., the synapse remained inhibitory), the ability of the presynaptic neuron to regulate its number of syn-
A

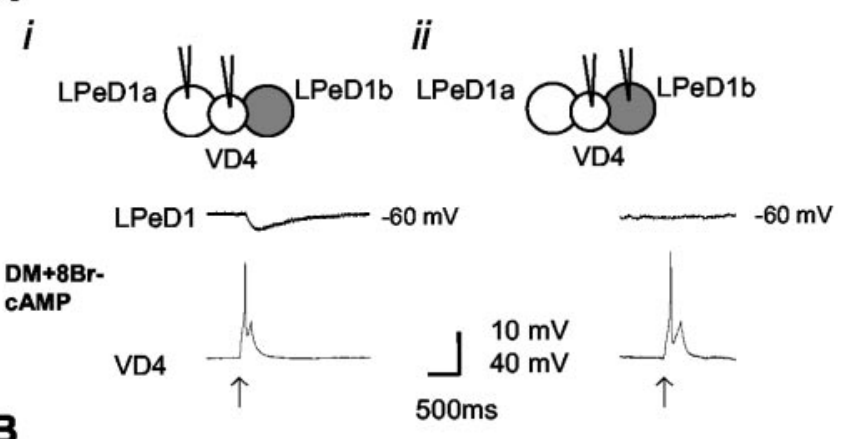

(12)

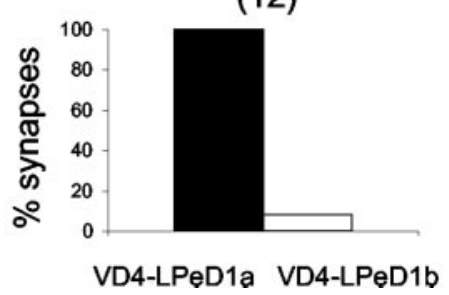

Figure 6. CAMP analogs rescue hyperinnervation in the absence of trophic factors. VD4LPeD1 triplets were paired in the absence of trophic factors and treated with 8Br-cAMP (100 $\mu \mathrm{M}) . A$, Pairwise intracellular recordings revealed that VD4 innervated only one LPeD1 target. $A i$, Evoked action potentials in VD4 induced 1:1 IPSPs in LPeD1a; in Aii, no postsynaptic response was detected in LPeD1b. B, Summary data of the percentage of LPeD1a and LPeD1b neurons that were innervated by VD4 neurons pretreated with PKA agonists. All postsynaptic responses were measured at a postsynaptic membrane potential of $-60 \mathrm{mV}$. Arrows indicate the injection of depolarizing current.

aptic connections was rescued, such that only one postsynaptic target was innervated (Fig. 6A). Evoked action potentials in VD4 induced 1:1 IPSPs in LPeD1a, but no postsynaptic response was detected in the second LPeD1b in $92 \%$ of the triplets treated with $8 \mathrm{Br}$-cAMP $(n=12)$ (Fig. $6 A, B)$. In $8 \mathrm{Br}$-cAMP-treated triplets, the size of unitary IPSPs in LPeD1a $(3.73 \pm 0.190)$ was not significantly different from that of untreated control pairs in DM $(4.14 \pm 0.154 \mathrm{mV})$. Together, these data demonstrate that $(1)$ cAMP-PKA-dependent pathways may not regulate the switch of synaptic phenotype from default inhibitory (Woodin et al., 1999) to excitatory; however, (2) presynaptic cAMP-PKA-dependent pathways may regulate synaptic efficacy and the number of synaptic outputs during synapse formation and synaptic plasticity. These data do not, however, rule out the possibility that the regulation of synaptic exclusion may also be a result of modulation of various existing components of the synaptic machinery.

\section{Discussion}

During development, the nature of signals that instruct any given presynaptic neuron that it has reached a full compliment of functional synapses and subsequently halt additional synaptogenesis remains largely unknown. Similarly, mechanisms that trim initial exuberant, supernumerary synapses so that the efficacy of synaptic transmission is maintained within a physiological range are also poorly understood. In this study, we have used a unique experimental approach to address the above issue and identified a novel mechanism by which a presynaptic neuron regulates both the efficacy of synaptic transmission and the formation of synapses at novel contact points. Specifically, dramatic changes in synaptic efficacy were observed when cAMP levels and PKA activity were perturbed pharmacologically such that increases in cAMP levels decreased synaptic efficacy, whereas inhibition of 
the cAMP-PKA pathway resulted in exuberant synapse formation and an enhancement of synaptic efficacy.

Because synaptic transmission still occurred in the presence of PKA agonists and antagonists, our data demonstrate that neither PKA agonists nor antagonists affected the ability of the presynaptic cell to release neurotransmitter. Although cAMP-PKA pathways regulate transmitter release in many systems (Kimura et al., 1998; Yoshihara et al., 2000; Okada et al., 2001), recent evidence suggests that exogenous application of PKA agonists does not affect evoked transmitter release at the invertebrate squid giant synapse, whereas PKA antagonists reversibly inhibit transmitter release (Hilfiker et al., 2001). Thus, we suggest a new role of the CAMP-PKA-dependent pathway in the presynaptic neuron: to provide a gain control over synaptic efficacy and de novo synapse formation with specific targets.

Both activity-dependent (Nguyen and Lichtman, 1996; Colman et al., 1997; Sanes and Lichtman, 1999; Ichise et al., 2000; Stellwagen and Shatz, 2002) and -independent (Nguyen and Lichtman, 1996; Nguyen et al., 1998) mechanisms have been proposed to account for the elimination of exuberant synapses. One attractive model is that active synapses receive trophic support at the expense of inactive synapses, which are consequently retracted (Nguyen et al., 1998; Sanes and Lichtman, 1999). Consistent with the role of trophic factors in synapse formation (Vicario-Abejon et al., 1998; Hamakawa et al., 1999; Woodin et al., 1999, 2002; Rico et al., 2002) and synaptic modulation (McAllister et al., 1999; Schinder and Poo, 2000; Woodin et al., 2002), we have demonstrated that brain-derived trophic factors are required for excitatory but not inhibitory synapse formation (Feng et al., 1997; Hamakawa et al., 1999; Woodin et al., 1999). Moreover, we have shown that in the absence of trophic factors, the second-messenger cascade mediating the exclusion signal fails to regulate the number of cells innervated by VD4. Because excitatory but not "default inhibitory" synapses (Woodin et al., 1999) were subjected to the exclusion signal, these data thus suggest that activity-dependent mechanisms may regulate exclusion of supernumerary synapses.

An activity-dependent regulation of synaptic exclusion would be consistent with our data, which show that given the equal opportunity, both LPeD1 cells could establish excitatory synapses with a single VD4 in CM during early stages of synapse formation (1-4 hr). Although VD4 formed synapses with both LPeD1 cells, the sum of total synaptic strength was maintained within a restricted range. This was accomplished by dividing the total synaptic efficacy between the two postsynaptic cells. At later stages of synapse maturation, however, one cell wins the full compliment of synaptic strength, whereas the other synapse is eliminated. This is reminiscent of synapse elimination at the neuromuscular junction, whereby supernumerary innervation persists as long as the inputs from all presynaptic neurons are of equal strength (Colman et al., 1997; Sanes and Lichtman, 1999). As soon as the balance is tipped in favor of one of the neurons, the weaker synapse is eliminated and the stronger prevails (Colman et al., 1997). The precise mechanisms that enable one LPeD1 cell to outcompete its counterpart for innervation by VD4 remain to be determined.

An alternative hypothesis suggests that the active synapse releases an unknown "poisonous" signal, which renders the inactive synapse nonfunctional and causes it to retract (Liu et al., 1994a, 1994b; Zoubine et al., 1996; Sanes and Lichtman, 1999). Our data presented in this study do not support this notion, because if either a VD4 or LPeD1a synapse were to poison the supernumerary LPeD1b cell, which was excluded or eliminated from the synaptogenic program, then it would have failed to receive innervation by the second VD4b (Fig. $1 E$ ). This most certainly was not the case, suggesting that the supernumerary synapses are actively and systematically eliminated by the presynaptic neuron.

Regulation of either synapse numbers or strength could explain changes that are observed during synaptic plasticity in both developing and adult nervous system (Martin et al., 1997a; Davis and Goodman, 1998a, 1998b; Lissin et al., 1998; Desai et al., 2002). However, difficulties in direct manipulations of individual presynaptic or postsynaptic elements at the single-synapse level, coupled with synaptic redundancy, have hindered additional analysis of changes that may account for synaptic scaling (Lissin et al., 1998; Watt et al., 2000; Desai et al., 2002) and neuronal plasticity (Davis and Goodman, 1998b; Davis et al., 1998; Marek et al., 2000; Davis and Bezprozvanny, 2001). For instance, genetic manipulations that perturb postsynaptic receptors at the Drosophila neuromuscular junction fail to register changes in the efficacy of synaptic transmission, because the presynaptic neurons counterbalance the postsynaptic deficit by either releasing more transmitter or increasing the size of its releasable quantal pool (Davis and Goodman, 1998b; Davis et al., 1998). Similarly, Drosophila mutants with hyperinnervated or hypoinnervated neuromuscular junction compensate for the perturbed presynaptic function by changing the number of postsynaptic receptors such that the efficacy of synaptic communication remains indistinguishable from the wild type (Davis and Goodman, 1998a, 1998b; Davis et al., 1998). In this study, we have demonstrated that when challenged simultaneously with two LPeD1 cells, a VD4 may innervate both cells but that this accommodation would not supersede the normal range of its total efficacy.

A recent study has demonstrated that in the absence of neuronal activity or during sensory deprivation, excitatory synaptic transmission in rat visual cortex is globally scaled up (Desai et al., 2002). Because this study had examined exclusively spontaneous events (i.e., miniature EPSC frequency and amplitude) in the absence of action potentials, it remains unclear whether synaptic potentials would also be similarly scaled. By examining synaptic transmission between defined presynaptic and postsynaptic cells, we have provided direct evidence that although synaptic events can indeed be scaled up, they do remain restricted within a preset physiological range. Specifically, a presynaptic neuron may provide gain control over its synaptic strength by scaling the number of its synapses (i.e., dual innervation with two LPeD1 cells); however, the sum of total synaptic efficacy remains within a preset range. Although a presynaptic neuron provides gain control over its synaptic strength, restrictions for a particular synaptic partner do not prevent this neuron from establishing a full compliment of synapses with other postsynaptic targets either in vivo or in vitro (Syed et al., 1990, 1992).

We propose that during synaptic development or plasticity at adult synapses, each synapse may activate a unique pathway in the presynaptic cell, such as the CAMP-PKA cascade described above, to regulate the number or strength of synapses. Additional mechanisms, such as those initiated by cell recognition, may act cooperatively to provide information about the source of the signal. Once the signal for detection of synaptic strength reaches a certain threshold, these messengers may in turn begin to instruct the presynaptic neuron to terminate new synapse formation with that particular postsynaptic neuron. Alternatively, they may also alter their existing synaptic machinery to maintain synaptic strength with a particular target within a restricted range. Thus, presynaptic cAMP-PKA activity serves to regulate the total 
number of synapses that a neuron would make, providing a precise balance between its input and output signals. Consistent with this model, we found that at an earlier time point during synapse formation, a VD4 simultaneously accommodates two LPeD1 neurons by scaling down its synaptic strength with each target. In these early synapses, the combined synaptic strength with both postsynaptic targets did not exceed the threshold for synaptic efficacy with a single postsynaptic target. Therefore, enhancing synaptic efficacy at one synaptic site comes at the expense of other synapses maintained by the presynaptic neuron. Our data raise an intriguing possibility that modulation of presynaptic cAMPPKA activity may underlie synaptic scaling as well as learning and memory.

\section{References}

Ahmari SE, Smith SJ (2002) Knowing a nascent synapse when you see it. Neuron 34:333-336.

Casadio A, Martin KC, Giustetto M, Zhu H, Chen M, Bartsch D, Bailey CH, Kandel ER (1999) A transient, neuron-wide form of CREB-mediated long-term facilitation can be stabilized at specific synapses by local protein synthesis. Cell 99:221-237.

Colledge M, Dean RA, Scott GK, Langeberg LK, Huganir RL, Scott JD (2000) Targeting of PKA to glutamate receptors through a MAGUK-AKAP complex. Neuron 27:107-119.

Colman H, Nabekura J, Lichtman JW (1997) Alterations in synaptic strength preceding axon withdrawal. Science 275:356-361.

Davis GW, Bezprozvanny I (2001) Maintaining the stability of neural function: a homeostatic hypothesis. Annu Rev Physiol 63:847-869.

Davis GW, Goodman CS (1998a) Synapse-specific control of synaptic efficacy at the terminals of a single neuron. Nature 392:82-86.

Davis GW, Goodman CS (1998b) Genetic analysis of synaptic development and plasticity: homeostatic regulation of synaptic efficacy. Curr Opin Neurobiol 8:149-156.

Davis GW, DiAntonio A, Petersen SA, Goodman CS (1998) Postsynaptic PKA controls quantal size and reveals a retrograde signal that regulates presynaptic transmitter release in Drosophila. Neuron 20:305-315.

Desai NS, Cudmore RH, Nelson SB, Turrigiano GG (2002) Critical periods for experience-dependent synaptic scaling in visual cortex. Nat Neurosci 5:783-789.

Diefenbach TJ, Guthrie PB, Kater SB (2000) Stimulus history alters behavioral response of neuronal growth cones. J Neurosci 20:1484-1494.

Fainzilber M, Smit AB, Syed NI, Wildering WC, Hermann PM, van der Schors RC, Jimenez C, Li KW, van Minnen J, Bulloch AG, Ibanez CF, Geraerts WP (1996) CRNF, a molluscan neurotrophic factor that interacts with the p75 neurotrophin receptor. Science 274:1540-1543.

Feng ZP, Klumperman J, Lukowiak K, Syed NI (1997) In vitro synaptogenesis between the somata of identified Lymnaea neurons requires protein synthesis but not extrinsic growth factors or substrate adhesion molecules. J Neurosci 17:7839-7849.

Garner CC, Zhai RG, Gundelfinger ED, Ziv NE (2002) Molecular mechanisms of CNS synaptogenesis. Trends Neurosci 25:243-251.

Hamakawa T, Woodin MA, Bjorgum MC, Painter SD, Takasaki M, Lukowiak K, Nagle GT, Syed NI (1999) Excitatory synaptogenesis between identified Lymnaea neurons requires extrinsic trophic factors and is mediated by receptor tyrosine kinases. J Neurosci 19:9306-9312.

Hermann PM, van Kesteren RE, Wildering WC, Painter SD, Reno JM, Smith JS, Kumar SB, Geraerts WP, Ericsson LH, Smit AB, Bulloch AG, Nagle GT (2000) Neurotrophic actions of a novel molluscan epidermal growth factor. J Neurosci 20:6355-6364.

Hilfiker S, Czernik AJ, Greengard P, Augustine GJ (2001) Tonically active protein kinase A regulates neurotransmitter release at the squid giant synapse. J Physiol (Lond) 531:141-146.

Huang YY, Li XC, Kandel ER (1994) cAMP contributes to mossy fiber LTP by initiating both a covalently mediated early phase and macromolecular synthesis-dependent late phase. Cell 79:69-79.

Ichise T, Kano M, Hashimoto K, Yanagihara D, Nakao K, Shigemoto R, Katsuki M, Aiba A (2000) mGluR1 in cerebellar Purkinje cells essential for long-term depression, synapse elimination, and motor coordination. Science 288:1832-1835.

Kim JH, Huganir RL (1999) Organization and regulation of proteins at synapses. Curr Opin Cell Biol 11:248-254.
Kimura S, Uchiyama S, Takahashi HE, Shibuki K (1998) cAMP-dependent long-term potentiation of nitric oxide release from cerebellar parallel fibers in rats. J Neurosci 18:8551-8558.

Leslie KR, Nelson SB, Turrigiano GG (2001) Postsynaptic depolarization scales quantal amplitude in cortical pyramidal neurons. J Neurosci 21:RC170(1-6).

Lissin DV, Gomperts SN, Carroll RC, Christine CW, Kalman D, Kitamura M, Hardy S, Nicoll RA, Malenka RC, von Zastrow M (1998) Activity differentially regulates the surface expression of synaptic AMPA and NMDA glutamate receptors. Proc Natl Acad Sci USA 95:7097-7102.

Liu Y, Fields RD, Festoff BW, Nelson PG (1994a) Proteolytic action of thrombin is required for electrical activity-dependent synapse reduction. Proc Natl Acad Sci USA 91:10300-10304.

Liu Y, Fields RD, Fitzgerald S, Festoff BW, Nelson PG (1994b) Proteolytic activity, synapse elimination, and the Hebb synapse. J Neurobiol 25:325-335.

Malinow R, Malenka RC (2002) AMPA receptor trafficking and synaptic plasticity. Annu Rev Neurosci 25:103-126.

Marek KW, Ng N, Fetter R, Smolik S, Goodman CS, Davis GW (2000) A genetic analysis of synaptic development: pre- and postsynaptic dCBP control transmitter release at the Drosophila NMJ. Neuron 25:537-547.

Martin KC, Kandel ER (1996) Cell adhesion molecules, CREB and the formation of new synaptic connections. Neuron 17:567-570.

Martin KC, Casadio A, Zhu H, Yaping E, Rose JC, Chen M, Bailey CH, Kandel ER (1997a) Synapse-specific, long-term facilitation of Aplysia sensory to motor synapses: a function for local protein synthesis in memory storage. Cell 91:927-938.

Martin KC, Michael D, Rose JC, Barad M, Casadio A, Zhu H, Kandel ER (1997b) MAP kinase translocates into the nucleus of the presynaptic cell and is required for long-term facilitation in Aplysia. Neuron 18:899-912.

McAllister AK, Katz LC, Lo DC (1999) Neurotrophins and synaptic plasticity. Annu Rev Neurosci 22:295-318.

Meems R, Munno DW, van Minnen J, Syed NI (2003) Synapse formation between isolated axons requires presynaptic soma and re-distribution of postsynaptic AChRs. J Neurophysiol, in press.

Mellor J, Nicoll RA, Schmitz D (2002) Mediation of hippocampal mossy fiber long-term potentiation by presynaptic Ih channels. Science 295:143-147.

Murthy VN, Schikorski T, Stevens CF, Zhu Y (2001) Inactivity produces increases in neurotransmitter release and synapse size. Neuron 32:673-682.

Nguyen QT, Lichtman JW (1996) Mechanism of synapse disassembly at the developing neuromuscular junction. Curr Opin Neurobiol 6:104-112.

Nguyen QT, Parsadanian AS, Snider WD, Lichtman JW (1998) Hyperinnervation of neuromuscular junctions caused by GDNF overexpression in muscle. Science 279:1725-1729.

Okada M, Nutt DJ, Murakami T, Zhu G, Kamata A, Kawata Y, Kaneko S (2001) Adenosine receptor subtypes modulate two major functional pathways for hippocampal serotonin release. J Neurosci 21:628-640.

Rico B, Xu B, Reichardt LF (2002) TrkB receptor signaling is required for establishment of GABAergic synapses in the cerebellum. Nat Neurosci 5:225-233.

Salin PA, Malenka RC, Nicoll RA (1996) Cyclic AMP mediates a presynaptic form of LTP at cerebellar parallel fiber synapses. Neuron 16:797-803.

Sanes JR, Lichtman JW (1999) Development of the vertebrate neuromuscular junction. Annu Rev Neurosci 22:389-442.

Schinder AF, Poo M (2000) The neurotrophin hypothesis for synaptic plasticity. Trends Neurosci 23:639-645.

Smit AB, Syed NI, Schaap D, van Minnen J, Klumperman J, Kits KS, Lodder $\mathrm{H}$, van der Schors RC, van Elk R, Sorgedrager B, Brejc K, Sixma TK, Geraerts WP (2001) A glia-derived acetylcholine-binding protein that modulates synaptic transmission. Nature 411:261-268.

Stellwagen D, Shatz CJ (2002) An instructive role for retinal waves in the development of retinogeniculate connectivity. Neuron 33:357-367.

Syed NI, Winlow W (1991) Coordination of locomotor and cardiorespiratory networks of Lymnaea stagnalis by a pair of identified interneurones. J Exp Biol 158:37-62.

Syed NI, Bulloch AG, Lukowiak K (1990) In vitro reconstruction of the respiratory central pattern generator of the mollusk Lymnaea. Science 250:282-285.

Syed NI, Ridgway RL, Lukowiak K, Bulloch AG (1992) Transplantation and 
functional integration of an identified respiratory interneuron in Lymnaea stagnalis. Neuron 8:767-774.

Syed NI, Zaidi H, Lovell P (1999) In vitro reconstruction of neuronal networks: a simple model system approach. In: Modern techniques in neuroscience research (Windhurst U, Johansson H, eds), pp 361-377. Berlin: Springer.

Turrigiano GG, Leslie KR, Desai NS, Rutherford LC, Nelson SB (1998) Activity-dependent scaling of quantal amplitude in neocortical neurons. Nature 391:892-896.

Vicario-Abejon C, Collin C, McKay RD, Segal M (1998) Neurotrophins induce formation of functional excitatory and inhibitory synapses between cultured hippocampal neurons. J Neurosci 18:7256-7271.

Watt AJ, van Rossum MC, MacLeod KM, Nelson SB, Turrigiano GG (2000) Activity coregulates quantal AMPA and NMDA currents at neocortical synapses. Neuron 26:659-670.

Weisskopf MG, Castillo PE, Zalutsky RA, Nicoll RA (1994) Mediation of hippocampal mossy fiber long-term potentiation by cyclic AMP. Science 265:1878-1882.
Woodin MA, Hamakawa T, Takasaki M, Lukowiak K, Syed NI (1999) Trophic factor-induced plasticity of synaptic connections between identified Lymnaea neurons. Learn Mem 6:307-316.

Woodin MA, Munno DW, Syed NI (2002) Trophic factor-induced excitatory synaptogenesis involves postsynaptic modulation of nicotinic acetylcholine receptors. J Neurosci 22:505-514.

Yoshihara M, Suzuki K, Kidokoro Y (2000) Two independent pathways mediated by cAMP and protein kinase A enhance spontaneous transmitter release at Drosophila neuromuscular junctions. J Neurosci 20:8315-8322.

Zhang JM, Li H, Liu B, Brull SJ (2002) Acute topical application of tumor necrosis factor alpha evokes protein kinase A-dependent responses in rat sensory neurons. J Neurophysiol 88:1387-1392.

Ziv NE, Garner CC (2001) Principles of glutamatergic synapse formation: seeing the forest for the trees. Curr Opin Neurobiol 11:536-543.

Zoubine MN, Ma JY, Smirnova IV, Citron BA, Festoff BW (1996) A molecular mechanism for synapse elimination: novel inhibition of locally generated thrombin delays synapse loss in neonatal mouse muscle. Dev Biol 179:447-457. 